\title{
Down Syndrome Phenotype in a Child with Partial Trisomy of Chromosome 21 and Paternally Derived Translocation t (20p; 21q)
}

Biaduń- Popławska Anna' ${ }^{1}$, Jamsheer Aleksander ${ }^{2}$, Henkelman Małgorzata', Tuziak Martyna ${ }^{3}$, Pietrzyk Aleksandra1 ${ }^{1}$, Piotrowski Krzysztof1*, Giżewska Maria', Walczak Mieczysław' ${ }^{1}$ and Zajączek Stanisław ${ }^{1}$

${ }^{1}$ Cytogenetic Unit, Department of Pathology, Pomeranian Medical University, Powstańców Wielkopolskich 72, 70-111 Szczecin, Poland

${ }^{2}$ Centre for Medical Genetics Genesis, Grudzieniec 4, 60-601 Poznań, Poland

${ }^{3}$ Clinic of Paediatrics, Endocrinology, Diabetology, Metabolic Diseases and Cardiology of the Developmental Age, Pomeranian Medical University, Unii Lubelskiej 1, 71252 Szczecin, Poland

\begin{abstract}
We present a case of the partial trisomy of chromosome 21 encompassing Down Syndrome Critical Region (DSCR) accompanied by 20p13 deletion. Patient's karyotype was determined as: 46, XY, der20t $(20,21)(21$ qter>21q22::20p13->21qter)pat. The boy presented nearly all typical features of Down syndrome but no specificsigns described in rare cases of patients with deletion 20p13. Microarray- CGH analysis has shown that trisomic segment contained DSCR whereas 20p deleted segment did not contain JAG1 gene. Clinical data of our patient was compared with the data available for other 48 patients carrying similar chromosomal microaberrations.
\end{abstract}

Keywords: Down syndrome critical region; Partial trisomy of chromosome 21; Down syndrome

\section{Introduction}

Down syndrome (DS) is the most frequent chromosomal cause of mental retardation. It usually results from the presence of additional copy of the entire chromosome 21 but in very few cases $(\sim 50$ worldwide) can be caused only by the partial trisomy of chromosome 21 , with a variable span of the duplicated fragment. Therefore, in the early 80 's of the twentieth century Epstein proposed that the clinical manifestation of DS may not only result from the pure chromosome 21 trisomy but also from the imbalances limited to a small fragment of the chromosome named Down Syndrome Critical Region (DSCR) [1]. DSCR was defined as responsible for: mental retardation, congenital heart anomalies of cushion type and some other minor (particularly facial) dysmorphic features. DSCR was mapped by two approaches, primarily by the "common minimal region of overlap" analysis of particularly rare cases and secondly by the analysis of the genetically engineered mice with trisomy of chromosome 16 corresponding to human trisomy of chromosome 21 [2].

Due to extremely rare incidence of patients with partial trisomy of chromosome 21 presenting full- blown DS phenotype we would like to present a boy with partial trisomy of chromosome 21 accompanied by small partial subtelomeric monosomy of chromosome 20, both resulting from the paternal balanced translocation $t(20,21)$. Similar cases have not been reported in the literature so far [3-6].

\section{Clinical Report}

We present a two-month-old boy who was being diagnosed on the grounds of clinical features suggesting DS. He was born as the $4^{\text {th }}$ pregnancy, $1^{\text {st }}$ delivery. The previous three pregnancies were spontaneously lost at 8-9 weeks of gestation. The child was born by caesarean section in the $39^{\text {th }}$ week of gestation due to foetal decelerations and oligohydroamnios and scored 8,9,10,10 points in Apgar score. His body weight was $2540 \mathrm{~g}$, length $46 \mathrm{~cm}$ and head circumference $32 \mathrm{~cm}$. All parameters were below the $3^{\text {rd }}$ percentile. Parents: a 24-year-old mother and 29 year-old father were Caucasian and non-consanguineous. Since the father had been adopted in early childhood, paternal family history was not available.

The first clinical evaluation was performed at the time of birth revealing: typical DS facial features, hypotonia, FOA and tricuspid valve insufficiency, which arose suspicion of DS. At two months the child was admitted to hospital due to respiratory infection, hypothyroidism and dysmorphic features, which led to subsequent, more detailed examinations. Heart ultrasound scan revealed FOA and tricuspid valve insufficiency of the $1^{\text {st }}$ degree without hemodynamic significance. Additionally, the child displayed hypotonia, strabismus and slight nystagmus. After the successful treatment of respiratory infection and introducing medications for hypothyroidism the child was evaluated by a specialist in clinical genetics. Standard karyotyping was performed, revealing additional unbalanced chromosomal material on short arm of chromosome 20. Upon genetic consultation the growth parameters were noted: body weight $-2730 \mathrm{~g}$, length- $51 \mathrm{~cm}$ and typical Down syndrome gestalt was observed including: large fontanel, sparse hair, low posterior hairline, prominent forehead, microophtalmia, flat occiput, small nose with low nasal bridge, small low-set ears, epicanthal folds, telecanthus, small mouth with thin lips, retrognathia and high arched palate, along with a short neck. We also noticed preaxial polysyndactyly and abnormal dermatoglyphs on his left hand but without the presence of the simian crease. Bilateral sandal gaps, right inguinal hernia and bilateral hydrocele of testis have also been found. The complete description of dysmorphic features observed in our patient is shown in Tables 1 and 2.

\section{Cytogenetic Analyses}

As mentioned above, the karyotype of peripheral blood lymphocytes showed additional chromosomal material located on short arm of chromosome 20 (Figure 1a). Striking DS appearance pointed further investigation into the long arm of chromosome 21 [7]. FISH analysis with painting probe for chromosome 21 (MP Biomedical;

*Corresponding author: Krzysztof Piotrowski, Cytogenetic Unit, Department of Pathology, Pomeranian Medical University, Powstańców Wielkopolskich 72, 70-11 Szczecin, Poland, Tel: +48 9146611 87, E-mail: olapietrzyk@wp.pl

Received May 28, 2014; Accepted September 25, 2014; Published October 05 2014

Citation: Anna BP, Aleksander J, Małgorzata H, Martyna T, Aleksandra P, et al (2014) Down Syndrome Phenotype in a Child with Partial Trisomy of Chromosome 21 and Paternally Derived Translocation t (20p; 21q). Gen Med (Los Angel) 2: 149. doi: 10.4172/2327-5146.1000149

Copyright: ( 2014 Anna BP, et al. This is an open-access article distributed under the terms of the Creative Commons Attribution License, which permits unrestricted use, distribution, and reproduction in any medium, provided the original author and source are credited. 